\title{
Standard planes for ultrasonographic examination of the portal system in dogs
}

\author{
Viktor Szatmári, DVM; Jan Rothuizen, DVM, PhD; George Voorhout, DVM, PhD
}

$\mathbf{P}^{2}$ ortosystemic shunting occurs when anomalous veins allow portal blood to directly enter the systemic veins without first flowing through the hepatic sinusoids. Portosystemic shunting is thought to be congenital if a single (or double) anomalous vein is present without concurrent portal hypertension, whereas it is considered to be acquired when collateral vessels form to compensate for sustained hepatic or prehepatic portal hypertension. ${ }^{1}$ Portosystemic shunting is suspected on the basis of history and clinical and laboratory findings; however, direct visualization of the aberrant vein or veins is essential for a definitive diagnosis and to rule out other conditions that can cause similar signs. ${ }^{2,3}$

Angiography is the most commonly used diagnostic modality in dogs suspected of having portosystemic shunting, as it allows differentiation of congenital versus acquired portosystemic shunting and can be used to distinguish intrahepatic congenital portosystemic shunts (CPSSs) from extrahepatic ones. ${ }^{4,5}$ However, portography is time-consuming and invasive. ${ }^{6}$ Similarly, although scintigraphy can be used to detect portosystemic shunting, it cannot be used to differentiate CPSSs from acquired portosystemic shunts (APSSs). ${ }^{7.8}$ Computed tomography and magnetic resonance imaging can provide anatomic information on abdominal blood vessels, ${ }^{9,10}$ but cannot give information on the direction of blood flow in those vessels. Moreover, both procedures are time consuming and require general anesthesia.

Ultrasonography has been used for diagnostic imaging of dogs with CPSSs since the $1980 \mathrm{~s}^{11}$ and has become popular because it is quick and noninvasive, does not require anesthesia or radiation, and allows for simultaneous evaluation of the abdominal organs. However, veterinarians tend to rely on the results of other or additional diagnostic procedures in dogs suspected to have portosystemic shunts because of the reported low sensitivity and specificity of ultrasonography. ${ }^{12}$ We believe that 1 reason for the reported low accuracy of ultrasonography in the diagnosis of portosystemic shunting is that no standardized protocol for examination of the portal system in dogs has been developed. Published reports ${ }^{2,11-14}$ typically focus only on some aspects of CPSSs and discuss the ultrasonographic features of APSSs only superficially or not at

From the Division of Diagnostic Imaging (Szatmári, Voorhout) and the Department of Clinical Sciences of Companion Animals (Rothuizen), Faculty of Veterinary Medicine, Utrecht University, Yalelaan 10, 3584 CM, Utrecht, The Netherlands.

Dr. Szatmári was supported by the Hungarian State Eötvös Scholarship.

Presented in part at the WSAVA-FECAVA-AVEPA Veterinary Congress in Granada, Spain, October 2002.

Address correspondence to Dr. Szatmári. all. The purpose of the present report, therefore, was to describe both a protocol for systematic ultrasonographic evaluation of the portal system in dogs and the ultrasonographic appearance of the portal system in healthy dogs.

\section{Description of the Technique}

Dogs were not sedated or anesthetized, and abdominal ultrasonography was performed with the dogs awake. Hair was clipped from the entire abdomen and over the last few ribs on the right side of the thorax, and contact gel was applied. First, routine B-mode real-time abdominal ultrasonography was performed. ${ }^{a}$ Then, the abdominal vasculature was evaluated in 7 standard planes by means of simultaneous B-mode and color flow Doppler imaging.

Parameters for color flow Doppler imaging were adjusted so that any vessel that was imaged was uniformly colored. ${ }^{15}$ In particular, the color gain was set so that color signals would be seen throughout the lumen of the vessel being examined, but not outside the vessel. The pulse repetition frequency (PRF) was also appropriately adjusted because a given PRF setting is able to detect only a range of velocities. If the PRF was set too high, slow flow might have been missed if the velocity of blood flow in the vessel of interest was lower than the lower limit of the velocity range for that PRF setting. If the blood flow velocity was higher than the upper limit of the velocity range, then aliasing artifacts were seen. Flow towards the transducer was coded red, and flow away from the transducer was coded blue. Higher flow velocities were coded with lighter shades of the appropriate color. If a velocity was higher than the upper limit of the velocity range, then the wrong color would be displayed (ie, the color that would be displayed would be the color indicating flow in the opposite direction). ${ }^{15}$

Plane 1: transverse intercostal plane with the dog in left lateral recumbency-The dog was positioned in left lateral recumbency, and the transducer was placed in 1 of the caudal right intercostal spaces. The aim was to find the intercostal space through which only the liver was seen, without the right kidney, and cross-sections of the aorta, caudal vena cava, and portal vein trunk could be visualized. If the right kidney appeared in the initial image, then the transducer was angled cranially or moved to a more cranial intercostal space. Alternatively, if air-containing lungs were seen, the transducer was angled caudally or moved to a more caudal intercostal space. If the portal vein could not be imaged because of gas in the gastrointestinal tract, the transducer was shifted dorsally in the same intercostal space and directed ventromedially. 
Plane 2: transverse intercostal plane with the dog in left lateral recumbency-Starting from plane 1 , the transducer was angled or slid cranially so that the portal vein could be followed to the point that a longitudinal image of the right portal branch appeared.

Plane 3: transverse intercostal plane with the dog in left lateral recumbency-Starting from plane 2, the transducer was gradually slid caudally, keeping the portal vein and caudal vena cava in the image, until the cranial mesenteric artery could be seen at its origin from the aorta. Scanning was performed first with Bmode ultrasonography, then was repeated with color flow Doppler imaging. The aim was to look for a direct connection between the portal vein and caudal vena cava or for a vessel that originated from the portal vein and had hepatofugal blood flow (ie, blood flow directed away from the portal vein).

Plane 4: longitudinal plane with the dog in left lateral recumbency-The transducer was placed immediately caudal to the last rib and directed craniomedially to obtain longitudinal images of the portal vein and the left and right portal branches. To find the portal vein, a longitudinal image of the aorta immediately ventral to the vertebrae was obtained. The transducer was then angled ventrally until the caudal vena cava became visible. Further ventral angulation of the transducer resulted in a longitudinal image of the portal vein at the point where the splenic vein entered the portal vein. Firm pressure on the transducer was often necessary to image the portal bifurcation.

In deep-chested dogs and large-breed dogs, the portal vein could not be visualized in this manner and an alternative approach was used. Specifically, starting from plane 1 , the transducer was rotated $90^{\circ}$ to obtain a longitudinal image of the portal vein.

Plane 5: longitudinal plane with the dog in dorsal recumbency-As an alternative for plane 4, the dog was placed in dorsal recumbency and then slightly tilted toward the sonologist. The right kidney and caudate liver lobe were imaged first. The transducer was then angled a bit ventromedially to image the caudal vena cava, then further ventromedially to image the portal vein. To image the portal bifurcation, the portal vein was followed cranially. Often, firm pressure on the transducer was needed to push gas-filled intestinal loops out of the way.

Plane 6: longitudinal plane with the dog in right lateral recumbency-With the dog in right lateral recumbency, the transducer was placed longitudinally immediately caudal to the last left rib and the portal vein was imaged at the hilus of the liver. Imaging the portal vein in this plane was rather difficult and was only necessary if an extrahepatic CPSS with right gastric vein origin was suspected on the basis of images obtained in plane 3 . The entire portal vein could not be visualized in plane 6 most of the time.

Plane 6 was also used to identify right gastric-caval shunts. To do this, the hepatic artery was followed from its origin to the liver because the hepatic artery would be expected to cross any right gastric-caval shunt that would be present. To find the hepatic artery, the celiac artery was imaged as it originated from the aorta cranial to the left kidney. ${ }^{15}$ The hepatic artery is the widest branch of the celiac artery, which runs cranially to the liver between the portal vein and the caudal vena cava. ${ }^{15}$ Color flow Doppler imaging was helpful in identifying the hepatic artery if gray scale resolution was insufficient to visualize this thin vessel. Color signals in the hepatic artery indicated higher flow velocity, compared with flow velocity in the caudal vena cava and portal vein

Plane 7: longitudinal plane with the dog in right lateral recumbency-The caudal vena cava was imaged in longitudinal section by placing the transducer immediately ventral to the lumbar vertebrae and caudal to the left kidney. Keeping the longitudinal image of the caudal vena cava, the transducer was slid cranially to image the left renal vein entering the caudal vena cava. With B-mode and color flow Doppler imaging, a search was performed for the left testicular or ovarian vein entering the left renal vein from caudally.

\section{Results}

Dogs-Twenty healthy adult Beagles of either sex were used to establish the normal ultrasonographic appearance of the portal system in dogs. In all dogs, serum bile acids and bilirubin concentrations and plasma alanine transaminase and alkaline phosphatase activities were within reference limits.

Plane 1-In plane 1, cross-sections of the aorta, caudal vena cava, and portal vein could be seen, with the aorta being most dorsal and the portal vein being most ventral (Fig 1). Cross-sectional areas of the 3 vessels were roughly equal; however, the caudal vena cava was often laterally compressed.

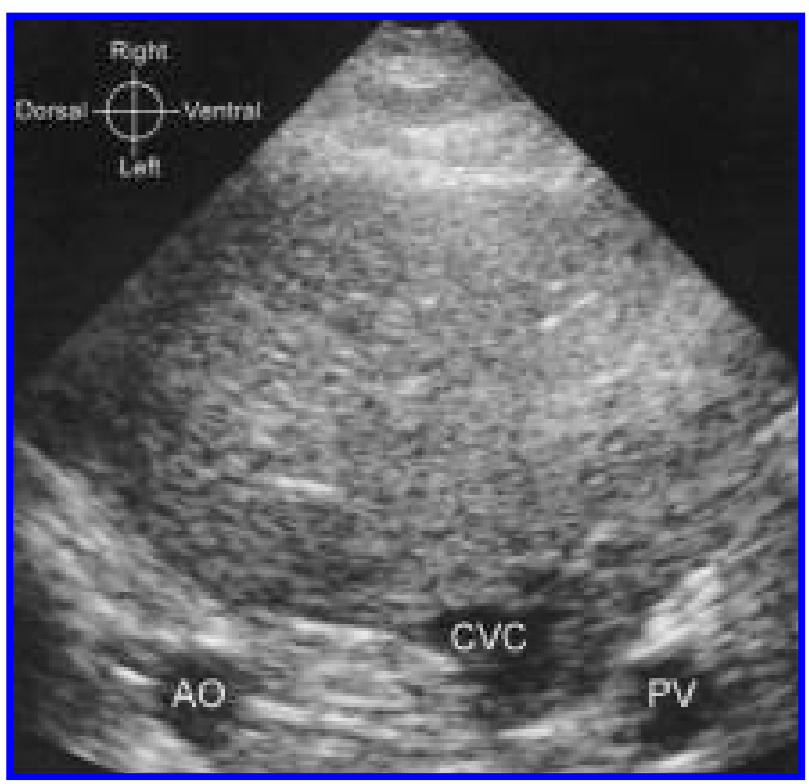

Figure 1-Ultrasonogram of the abdomen of a healthy male adult Beagle. The dog was in left lateral recumbency, and the transducer was placed in the last right intercostal space and oriented transversely (plane 1). This was the starting point for a systematic ultrasonographic examination of the portal system. From dorsal to ventral, cross-sections of the aorta (AO), caudal vena cava (CVC), and portal vein (PV) can be seen. 


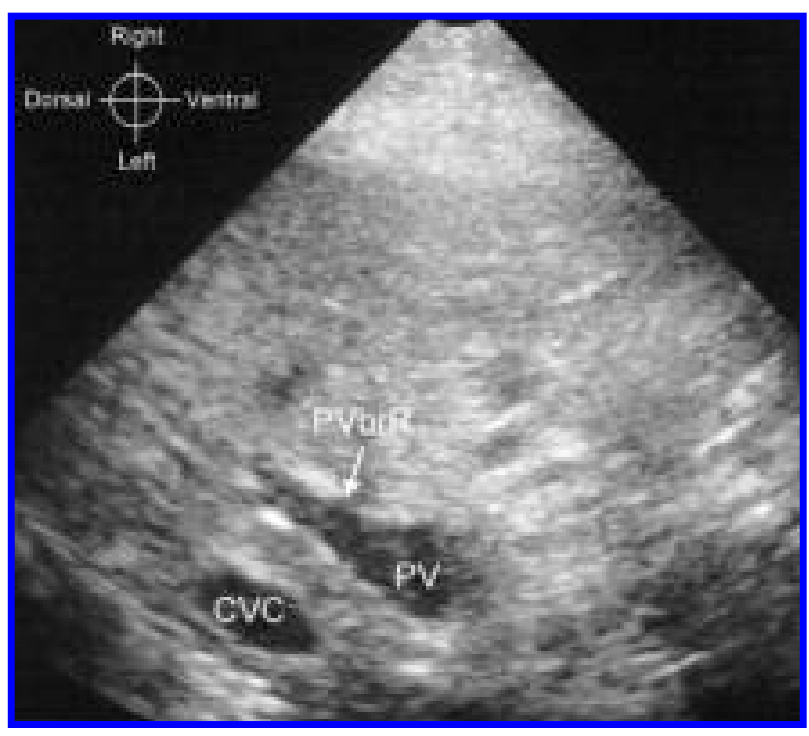

Figure 2-Ultrasonogram of the abdomen of a healthy male adult Beagle. The dog was in left lateral recumbency, and the transducer was oriented transversely and placed in a more cranial right intercostal space, compared with the orientation in Figure 1 (plane 2). The right portal branch (PVbrR) can be seen at its origin from the PV. The right portal branch is thinner than the PV and becomes gradually thinner towards the periphery because of ramification. Notice that the right portal branch runs dorsolaterally and to the right. See Figure 1 for remainder of key.

Plane 2-The right portal branch was consistently found as a well-defined vein originating from the portal vein and running dorsolaterally to the right while becoming gradually thinner because of ramification (Fig 2).

Plane 3-Immediately caudal to the portal bifurcation, the gastroduodenal vein could occasionally be imaged as it entered the ventral aspect of the portal vein from the right; however, the gas-filled descending duodenum often hindered its visualization. Sliding the transducer further caudally, the splenic vein could be seen entering the left aspect of the portal vein from a ventrolateral direction. Color flow Doppler imaging revealed hepatopetal flow in the splenic vein. Slightly caudal to this point, the origins of the celiac and cranial mesenteric arteries from the aorta could be seen. Both arteries ran ventrally.

Plane 4-The splenic vein was seen to enter the portal vein from a caudolateral direction from the left (Fig 3). Following the portal vein cranially, the portal bifurcation was seen with the wider left and thinner right portal branches. Both branches became gradually thinner towards the periphery (Fig 4).

Plane 5-The findings were the same as described for plane 4, but in some instances, plane 5 allowed better visualization of the portal vein and better incidence angles for color flow Doppler imaging.

Plane 6-A portion of the portal vein could be seen from this plane. The right gastric vein was so thin that it could not be visualized ultrasonographically.

Plane 7-Caudal to the left kidney, the aorta and caudal vena cava could be seen; the aorta was located

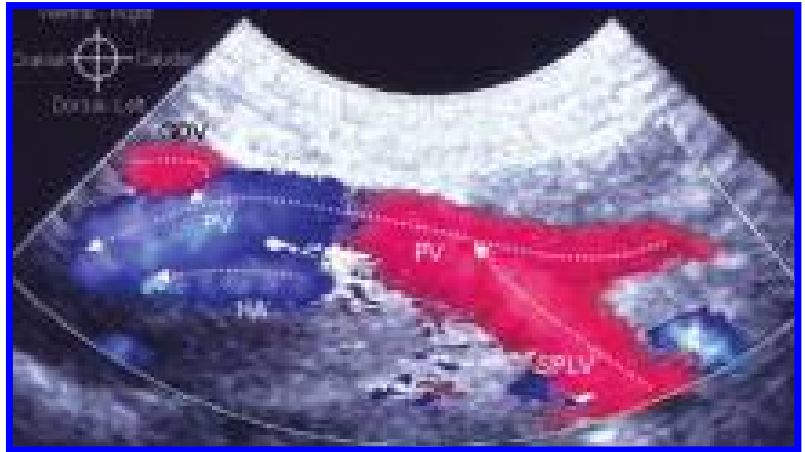

Figure 3-Color flow Doppler ultrasonogram of the PV in a dog. The diameter of the PV is uniform along its whole length. The transducer is placed longitudinally, caudal to the last right rib, with the dog in left lateral recumbency (plane 4). GDV = Gastroduodenal vein. SPLV = Splenic vein. $\mathrm{HA}=$ Hepatic artery.

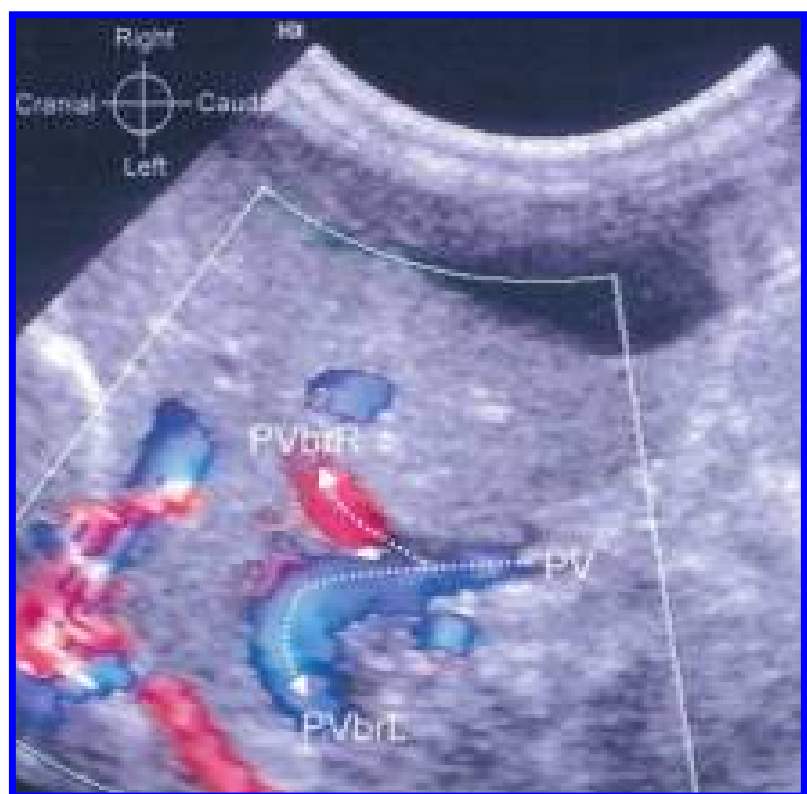

Figure 4-Color flow Doppler ultrasonogram of the portal bifurcation in a dog. Notice that the left portal branch (PVbrL) is wider than the right (PVbrR) and that both become gradually thinner toward the periphery. The image was obtained with the dog in left lateral recumbency and the transducer in the right flank (plane 4).

more to the left. The left testicular or ovarian vein could never be visualized. The left renal artery (occasionally double) ran adjacent to the left renal vein and could be recognized even on gray scale images by its smaller diameter and pulsation.

\section{Discussion}

Previous studies ${ }^{2,13,14}$ that involved ultrasonographic differentiation of CPSSs from other diseases reported sensitivity of $74 \%$ to $95 \%$ and specificity of $57 \%$ to $100 \%$. However, we believe that in 2 of these studies, some extrahepatic CPSS were not recognized because only a right intercostal approach was used. For example, in a study ${ }^{16}$ of 90 dogs with hyperammonemia secondary to CPSSs, APSSs, or urea cycle enzyme deficiency, we found that congenital extrahepatic right gastric-caval shunts cannot be readily identified from a right intercostal approach because visualization of the 
cranial shunt loop requires that the dog be in right lateral recumbency, whereas visualization of the caudal shunt loop requires examination with the transducer orientated longitudinally in the right flank. Thus, it is possible that extra- and intrahepatic CPSSs were not identified in previous studies because evaluation of the abdominal vasculature was not systematic and the right portal branch was not regularly imaged. Indeed, in our experience, congenital extrahepatic spleniccaval shunts seem to be surrounded by liver when they are scanned via a right intercostal approach.

In a study ${ }^{16}$ of 90 dogs with hyperammonemia, we found that the present protocol for systematic ultrasonographic evaluation of the portal system was effective in identifying CPSSs and APSSs and ruling out portosystemic shunting in dogs with urea cycle enzyme deficiencies. In our experience, however, high accuracy also required that in addition to use of the scanning protocol, a high-quality ultrasound machine with excellent gray scale resolution was needed. Use of color flow Doppler imaging was not essential; however, it greatly facilitated the diagnosis of abdominal vascular diseases.

Even if the scanning protocol described in the present report is used, accurate ultrasonographic recognition of CPSSs is possible only if the anomalous vein is followed from its origin to its termination. Finding the point where a CPSS enters the caudal vena cava may be easier than finding the origin of a $\mathrm{CPSS}^{14}$; however, finding a vein that enters the caudal vena cava does not mean that it is a CPSS because several veins normally enter the caudal vena cava. If the origin and course of a suspected CPSS is not visualized, a normal right renal or phrenicoabdominal vein could be mistaken for a CPSS. In contrast, if a vein that originates from the portal vein or a portal tributary has hepatofugal blood flow, it is surely an extrahepatic CPSS or an APSS because in normal animals, veins only enter the portal vein and do not originate from it. ${ }^{13}$

In our opinion, even with the present protocol, identifying left divisional intrahepatic CPSS will be difficult because such shunts run close to the diaphragm, making visualization via the intercostal spaces impossible in most instances. Scanning via the right flank requires hard transducer pressure because of the cranial displacement of the intestines secondary to the small liver, and this much pressure on the transducer causes discomfort to the dog.

Diagnosing APSSs ultrasonographically requires a different approach than diagnosing CPSSs because the collateral veins only occasionally arise directly from the portal vein. Moreover, these collateral vessels are thin and tortuous and often hidden among the intestines. Thus, their origins and courses can only rarely be revealed. In our study ${ }^{16}$ of dogs with hyperammonemia, however, dilatation of the left testicular or ovarian vein was found to be a highly specific and sensitive indicator of APSSs, and its ultrasonographic visualization was easy.
In sum, we believe that the described scanning protocol will be useful in the ultrasonographic evaluation of dogs for CPSSs and APSSs. In dogs with portosystemic shunting, not only should anomalous veins be visible with this technique, but the abdominal vasculature should look quite different from the vasculature in healthy dogs. The 4 sites that should always be imaged if a portal vein anomaly is suspected are the right portal branch, the left testicular or ovarian vein, the portal vein at the level of the celiac artery (splenic vein), and the portal vein immediately caudal to the portal bifurcation (right gastric vein and gastroduodenal vein).

${ }^{a}$ ATL HDI 3000, Advanced Technology Laboratories Ultrasound, Philips Medical Systems, Woerden, The Netherlands.

\section{References}

1. van den Ingh TSGAM, Rothuizen J, Meyer HP. Circulatory disorders of the liver in dogs and cats. Vet $\mathcal{Q}$ 1995;17:70-76.

2. Tiemessen I, Rothuizen J, Voorhout G. Ultrasonography in the diagnosis of congenital portosystemic shunts in dogs. Vet $\mathcal{Q}$ $1995 ; 17: 50-53$.

3. Strombeck DR, Meyer DJ, Freedland RA. Hyperammonemia due to a urea cycle enzyme deficiency in two dogs. I Am Vet Med Assoc 1975;166:1109-1111.

4. Ewing GO, Suter PF, Bailey CS. Hepatic insufficiency associated with congenital anomalies of the portal vein in dogs. J Am Anim Hosp Assoc 1974;10:463-476.

5. Suter PF. Portal vein anomalies in the dog: their angiographic diagnosis. IAm Vet Radiol Soc 1975;16:84-97.

6. Breznock EM. Surgical manipulation of portosystemic

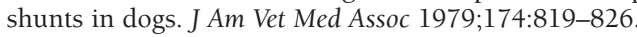

7. Meyer HP, Rothuizen J, van den Brom WE, et al. Quantitation of portosystemic shunting in dogs by ultrasound-guided injection of 99MTc-macroaggregates into a splenic vein. Res Vet Sci 1994;57:58-62.

8. Koblik PD, Hornof WJ. Transcolonic sodium pertechnetate Tc $99 \mathrm{~m}$ scintigraphy for diagnosis of macrovascular portosystemic shunts in dogs, cats, and potbellied pigs: 176 cases (1988-1992). I Am Vet Med Assoc 1995;207:729-733.

9. Kleiter M, Henninger W, Hirt R, et al. Portosystemic shunt in a dog-computed tomography as a successful imaging method. Wien Tierarztl Monatssch 1999;86:64-70.

10. Seguin B, Tobias KM, Gavin PR, et al. Use of magnetic resonance angiography for diagnosis of portosystemic shunts in dogs. Vet Radiol Ultrasound 1999;40:251-258.

11. Wrigley RH, Konde LJ, Park RD, et al. Ultrasonographic diagnosis of portacaval shunts in young dogs. I Am Vet Med Assoc 1987;191:421-424.

12. Nyland TG, Mattoon JS, Herrgesell EJ, et al. Liver. In: Nyland TG, Mattoon JS, eds. Small animal diagnostic ultrasound. 2nd ed. Philadelphia: WB Saunders Co, 2002;93-127.

13. Holt DE, Schelling CG, Saunders HM, et al. Correlation of ultrasonographic findings with surgical, portographic, and necropsy findings in dogs and cats with portosystemic shunts: 63 cases (1987-1993). LAm Vet Med Assoc 1995;207:1190-1193.

14. Lamb CR. Ultrasonographic diagnosis of congenital portosystemic shunts in dogs: results of a prospective study. Vet Radiol Ultrasound 1996;37:281-288.

15. Szatmári V, Sótonyi P, Vörös K. Normal duplex Doppler waveforms of the major abdominal blood vessels in dogs: a review. Vet Radiol Ultrasound 2001;42:93-107.

16. Szatmári V, Rothuizen J, van den Ingh TSGAM, et al. Ultrasonographic findings in dogs with hyperammonemia: 90 cases (2000-2002). L Am Vet Med Assoc 2004;224:717-727. 